\title{
DENSITY OF MATTER IN THE GALACTIC DISK
}

\author{
C. FLYNN
}

NORDITA, Copenhagen, Denmark

\author{
AND \\ B. FUCHS \\ Astronomisches Rechen-Institut Heidelberg, Germany
}

A long standing issue in Astronomy is the amount of unseen matter in the Galactic disk. This has been addressed many times since Oort (1960) showed that about half the local disk mass is in unseen material. Oort's method infers the disk mass via the vertical change in density and velocity dispersion of appropriate tracer stars. Recently, Kuijken and Gilmore (1989) analysed a sample of K dwarfs at the South Galactic Pole and found in fact no compelling evidence for dark matter in the disk - a conclusion also reached by Bienaymé, Robin and Crézé (1987) based on faint star count data. However, Bahcall, Flynn and Gould (BFG, 1992), analysed K giants at the South Galactic Pole, and while they corroborated the Oort result, they could state at only the $85 \%$ confidence level that at least some disk matter is 'missing'. Here we describe the Flynn and Fuchs (1994) analysis of a sample of nearby K-giants, which they combine with the BFG sample. The combined sample can be well fit without dark matter in the disk. The fit can be improved using a small amount of disk dark matter: our best fit has a disk surface density of $\Sigma_{\odot}=53 \pm 13 \mathcal{M}_{\odot} \mathrm{pc}^{-2}$, compared to $\Sigma_{\odot}$ $=49 \pm 9 \mathcal{M}_{\odot} \mathrm{pc}^{-2}$ for the known disk matter. We conclude the disk is relatively light and the dark halo makes a significant contribution to the rotation curve at the sun.

\section{References}

Bahcall,J.N.: 1984, ApJ 276, 156

Bahcall,J.N., Flynn,C., Gould,A.: 1992, ApJ 389, 234 (BFG)

Bienaymé, O., Robin A., Crézé, M. 1987. A\&A 180, 94

Flynn,C., Fuchs,B.: 1994, MNRAS in press

Kuijken,K., Gilmore,G.: 1989, MNRAS 239, 571

Oort,J.H.,1960.Bull.Astr.Inst.Netherlands,15,45 